\title{
1947-1957. Dixième anniversaire de « l'engagement » ou Monsieur de Norpois et la « littérature-banane»
}

\section{Claude Simon}

\section{(2) OpenEdition \\ Journals}

Édition électronique

URL : http://journals.openedition.org/ccs/575

DOI : $10.4000 /$ ccs. 575

ISSN : 2558-782X

Éditeur :

Presses universitaires de Rennes, Association des lecteurs de Claude Simon

\section{Édition imprimée}

Date de publication : 30 avril 2007

Pagination : 131-135

ISBN : 9782354120122

ISSN : $1774-9425$

\section{Référence électronique}

Claude Simon, « 1947-1957. Dixième anniversaire de « l'engagement » ou Monsieur de Norpois et la "littérature-banane » », Cahiers Claude Simon [En ligne], 3 | 2007, mis en ligne le 21 septembre 2017, consulté le 07 mai 2019. URL : http://journals.openedition.org/ccs/575; DOI : 10.4000/ccs.575 


\title{
1947-1957
}

Dixième anniversaire de "l'engagement » ou Monsieur de Norpois et la "littérature-banane »

\author{
par Claude SIMON
}

(inédit)

« Il entre dans la notion de l'homme universel cette caractéristique essentielle qu'il n'est engagé dans aucune époque particulière, et qu'il ne s'émeut ni plus ni moins sur le sort des nègres de la Louisiane que sur celui des esclaves romains de Spartacus », écrit Sartre avec un dégoût non dissimulé dans Qu'est-ce que la littérature?

Cinquante ans plus tôt environ, le jeune Proust qui, au grand effroi de ses parents, sentait poindre sa vocation d'écrivain, entendait tomber de la bouche de M. de Norpois le discours suivant : « Dans un temps comme le nôtre où la complexité croissante de la vie laisse à peine le temps de lire, où la carte de l'Europe a subi des remaniements profonds et est à la veille d'en subir de plus grands encore peut-être, où tant de problèmes menaçants et nouveaux se posent partout, vous m'accorderez qu'on a le droit de demander à un écrivain d'être autre chose qu'un bel esprit qui nous fait oublier dans des discussions oiseuses et byzantines sur des mérites de pure forme que nous pouvons être envahis d'un instant à l'autre par un double flot de Barbares, ceux du dehors et ceux du dedans. Je sais que c'est blasphémer la Sacro-Sainte École de ce que ces messieurs 
appellent l'Art pour l'Art, mais à notre époque, il y a des tâches plus urgentes que d'agencer des mots d'une façon harmonieuse [...]. Toutes ces chinoiseries de forme, toutes ces subtilités de mandarin déliquescent me semblent bien vaines. »

C'est ainsi que Proust était invité sans équivoque à « s'engager». Avec, en moins, le jargon philosophique et, en plus, une louable concision, il est assez troublant de trouver dans cette diatribe tous les thèmes que développera plus tard l'inventeur de l'existentialisme. L'ambassadeur fin de siècle et le philosophe du demi-siècle semblent en effet partager, en même temps que le mépris de l'Art pour l'Art et des Mandarins, d'identiques préoccupations, fort nobles d'ailleurs (souci du « devoir», de " l'utilité » de l'écrivain, et de cet « esprit de sérieux » bien connu par ailleurs des lecteurs de petites annonces dans lesquelles la peur lancinante d'être dupe fait multiplier comme un leitmotiv par les dames en quête de l'âme-sœur la célèbre formule : "Si pas ser. s'abst. ») et auxquelles ils voient des solutions communes.

«Dans un temps comme le nôtre », dit M. de Norpois, posant ainsi d'emblée le problème de l'historicité cher à Sartre qui évoquant sans cesse « la pression de l'histoire », prône « l'appel singulier et daté qu'un homme [lance] en acceptant de s'historialiser ». Car, pour lui aussi, la question d'écrire se pose en termes élevés de morale : «L'écrivain est un homme qui doit répondre à une certaine demande. »- «On a le droit de demander à un écrivain », dit Norpois. Quant à cette « demande » elle-même, quelle est-elle ? Norpois la formule sans ambages : « À notre époque il y a des tâches plus urgentes que d'agencer des mots d'une façon harmonieuse. » - «On ne doit pas s'attarder à faire chanter le violoncelle », approuve Sartre qui, stigmatisant précisément ce $\mathrm{XIX}^{\mathrm{e}}$ siècle dont la production littéraire indigne aussi Norpois, ajoute : "La somptuosité des moyens d'expression qu'il a découverts ne doit pas nous faire oublier qu'il a trahi la littérature. »

Naturellement, on ne saurait faire grief à nos deux penseurs de soucis d'une aussi haute inspiration. Nous les éprouvons tous plus ou moins, et peu d'hommes, sauf quelques pauvres malades mentaux, peuvent sans faiblir soutenir devant eux-mêmes (en faire parade est autre chose) une posture d'immoraliste. 
Mais ce qui semble encore rapprocher Sartre et Norpois en les différenciant des vulgaires «moralistes" que nous sommes tous, c'est ce curieux sentiment d'une catastrophe imminente qui les obsède tous deux, comme si une sorte de seconde vue leur donnait, du monde, une vision angoissée et apocalyptique. C'est tout juste si « demain » existe, " après demain » non. Il y a " urgence », dit Norpois - «On ne doit pas s'attarder», dit Sartre, et c'est donc tout de suite que l'action de l'écrivain doit se faire sentir. En un mot, pour Norpois, et selon la brillante formule de Sartre, «les ouvrages de l'esprit, pareillement aux bananes, doivent se consommer sur place».

On sait l'artifice, le tour de passe-passe par lequel Sartre justifie ce postulat : l'auteur d'une œuvre « inconsommable sur place» n'a et n'aura jamais qu'un vain public de «spécialistes». Croire que ce public puisse, dans le futur, s'élargir, c'est « le mythe de la gloire».

Répondant indirectement à M. de Norpois, et, par avance, à Sartre, Proust avait pourtant analysé d'une façon géniale le mécanisme complexe de la lente pénétration du grand public par la culture (ce phénomène qui fait s'arrêter aujourd'hui avec respect devant les toiles de Cézanne le même père de famille qui menait hier sa progéniture s'esclaffer chez Vollard et place dans toute bibliothèque « qui se respecte » un exemplaire de Madame Bovary)

«Toute nouveauté, écrit-il, ayant pour condition l'élimination préalable du poncif auquel nous étions habitués et qui nous semblait la réalité même, toute conversation neuve, aussi bien que toute peinture, toute musique originale, paraîtra toujours alambiquée et fatigante [...], chacun appelant idées claires celles qui sont au même degré de confusion que les siennes propres. Au fond les anciennes formes de langage avaient été elles aussi autrefois ces images difficiles à suivre quand l'auditeur ne connaissait pas encore l'univers qu'elles peignaient. Mais depuis longtemps on se figure que c'était l'univers réel et on se repose sur lui [...]. Mais moins décevants que la vie, ces grands chefs-d'œuvre ne commencent pas par nous don- 
ner ce qu'ils ont de meilleur [...]. Le temps qu'il faut à un individu [...] pour pénétrer une œuvre un peu profonde n'est que le raccourci et comme le symbole des années, des siècles parfois, qui s'écoulent avant que le public puisse aimer un chef-d'œuvre vraiment nouveau. [...] Ce qui est cause qu'une œuvre de génie est difficilement admirée tout de suite, c'est que celui qui l'a écrite est extraordinaire, que peu de gens lui ressemblent. C'est son œuvre elle-même qui, en fécondant les rares esprits capables de la comprendre, les fera croître et multiplier [...]. Ce que l'on appelle la postérité, c'est la postérité de l'œuvre. »

Peut-être, lorsqu'il écrivit Qu'est-ce que la littérature Sartre ne connaissait-il pas ces lignes de Proust ? Il est vrai que, de celui-ci, il dit avec superbe qu'il offre l'exemple le plus achevé de cette «psychologie intellectualiste » et, qu'à ce titre, il croit «à l'existence de passions universelles dont le mécanisme ne varie pas sensiblement quand on modifie les caractères sexuels, les conditions sociales, la nation ou l'époque des individus qui les ressentent $»$.

Resterait à expliquer comment ce "pédéraste » qui, en " croyant à l'existence de passions universelles » s'est fait «le complice de la propagande bourgeoise », a pu prophétiquement mettre dans la bouche d'un de ses personnages les propos que tiendra presque mot pour mot, un demi-siècle plus tard, un philosophe aux idées "révolutionnaires" (car il n'est même pas sûr que ces "Barbares du dehors et du dedans » tant redoutés par M. de Norpois ne soient pas, au fond, les mêmes que ceux que Sartre vilipende : que l'on se rappelle en effet l'accusation de germanisme lancée en 1914 par la critique traditionaliste et réactionnaire contre les cubistes et leur marchand Kahnweiler et, d'autre part, le rôle joué par Dada et de nombreux intellectuels venus de l'Est dans ce mouvement surréaliste désigné par le théoricien de l'engagement comme la quintessence de la trahison littéraire...).

Quant à savoir pourquoi, à tant d'années d'intervalle et malgré la modification des caractères sexuels, des conditions sociales, etc., un vieil ambassadeur royaliste et colossalement riche a pu avoir la 
même conception de la littérature qu'un professeur de philosophie aux idées « avancées », c'est là une question que n'ont probablement pas encore fini d'élucider bon nombre de jeunes écrivains qui, après avoir écouté avec recueillement les conseils de Sartre se sont sentis pénétrés par le doute jusqu'au tréfonds d'eux-mêmes, comme le jeune Proust passionnément attaché à retrouver dans un fugace parfum « la matière éternelle et commune » et pensant avec mélancolie, tandis qu'il attendait Françoise contre le mur humide du petit pavillon treillagé des Champs-Elysées : «Il me sembla que je méritais vraiment le dédain de $\mathrm{M}$. de Norpois ; j'avais préféré jusqu'ici à tous les écrivains celui qu'il appelait un simple "joueur de flûte" et une véritable exaltation m'avait été communiquée, non par quelque idée importante, mais par une odeur de moisi. » 